Pathologe 2009 · 30:4-5

DOI 10.1007/s00292-008-1098-8

Online publiziert: 5. Februar 2009

(c) Springer Medizin Verlag 2009

\author{
W. Böcker · T. Decker \\ Gerhard-Domagk-Institut für Pathologie, Münster
}

\title{
Aktuelle Aspekte der Mammapathologie
}

\section{Zellbiologie}

Diskurs, sich ihres Gegenstands beschreibend und erklärend zu vergewissern. Auffällig ist, dass die Pathologen sich dies in der Vergangenheit unmittelbar zutrauten, während wir heute zu demselben Zweck ganz verschiedene Disziplinen und/oder Methoden zusammenführen müssen: in der Praxis z.B. Radiologie, Pathologie und Gynäkologie, in der Wissenschaft z. B. Pathologie und Molekularbiologie. Diese allgemeinen Bemerkungen gelten somit auch und insbesondere für die Mammapathologie.

Die Mammapathologie stellt eine Vielfalt heterogener Erkrankungen dar, die sich hinsichtlich ihrer klinischen Präsentation, Histologie sowie klinischen Verläufe unterscheiden. Obwohl die geltenden histopathologischen Klassifikationssysteme heute nach wie vor auf deskriptiven Entitäten basieren, sind sie in neuerer Zeit durch zellbiologische und molekulare Konzepte ergänzt worden. Dies hat zu einer Vertiefung und Erweiterung des Verständnisses sowohl des normalen Brustdrüsenepithels als auch ihrer proliferativen Erkrankungen geführt. Diese Erkenntnisse werden z. T. auch bereits im differenzialdiagnostischen Methodenspektrum proliferierender Erkrankungen berücksichtigt. Darüber hinaus haben die Fortschritte in der Bildgebung und interventionellen Radiologie mit den sich daraus ergebenden Möglichkeiten zum Mammographiescreening den Aufgabenbereich des Mammapathologen erheblich erweitert, vergleichbar mit der Einführung der Endoskopie für den gastrointestinalen Pathologen. In dem vorliegenden Schwerpunktheft sollen drei Aspekte der heutigen Mammapathologie in mehreren Beiträgen beleuchtet werden.
Die Anwendung basaler ( $\left.\mathrm{CK}_{5} / 14 / 17\right)$ und luminaler Zytokeratine $\left(\mathrm{CK}_{7} / 8 / 18\right)$ sowie myoepithelialer Marker in der Immunhistochemie bzw. -fluoreszenzmikroskopie mit der gleichzeitigen In-situDarstellung mehrerer Antigene hat im letzten Jahrzehnt zu einem neuen zellbiologischen Progenitorzellmodell des Brustdrüsenepithels geführt. Das Modell postuliert CK5/14-positive Progenitorzellen, die sich über Zwischenstufen zu CK8/18positiven glandulären oder sm-Aktinpositiven myoepithelialen Zellen differenzieren. Mit Hilfe der Immunhistochemie kann der Pathologe erstmals definierte Subtypen des normalen Mammaepithels in ein Ordnungssystem eingliedern. Da dieses Modell eine wichtige Grundlage für das Verständnis proliferativer Mammaerkrankungen darstellt, werden Böcker et al. in dem Beitrag zur Anatomie der Brustdrüse diese Problematik diskutieren.

Bei den benignen proliferativen Prozessen finden sich auffallende Parallelen in der zellulären Zusammensetzung zum normalen Brustdrüsenepithel. Bei den malignen Prozessen ist die Ähnlichkeit zu bestimmten Subtypen des normalen Mammaepithels so charakteristisch, dass sich daraus histogenetische Beziehungen vermuten lassen. So bietet das Modell erstmals eine Erklärung für das Vorkommen luminaler und basalzelliger maligner Tumoren in diesem Organ. Die zellbiologischen Charakteristika stellen in jedem Fall eine rationale Basis für die Möglichkeit dar, die verschiedenen proliferativen Läsionen immunhistochemisch zu unterscheiden (z. B. die einfache duktale Hyperplasie und die duktalen und lobulären Neoplasien etc.). In ihrem Beitrag zur Im- munhistochemie werden Böcker et al. auf die differenzialdiagnostischen Aspekte proliferativer Mammaläsionen eingehen.

\section{Molekulare Mechanismen}

Aktuell erweitern und modifizieren die Ergebnisse moderner molekularer Techniken unser Verständnis des Mammakarzinoms. Bereits frühe Daten der komparativen genomischen Hybridisierung (CGH) und der Mikrosatelliteninstabilität haben gezeigt, dass die Karzinomentstehung auf verschiedenen Entwicklungswegen („low-grade“ und „high-grade pathway") verlaufen kann und dass die frühesten Läsionen (flache epitheliale Atypie und atypische duktale Hyperplasie) bereits mit molekularen Veränderungen der Zellen einhergehen, wie sie dann später auch in fortgeschritteneren nichtinvasiven und invasiven Karzinomen zu finden sind.

Unter den molekularen Methoden erlangte die Genexpressionsanalyse auf Microarraybasis in letzter Zeit die größte Aufmerksamkeit. Diese Methode wurde mit Erfolg benutzt, um eine neue molekulare Taxonomie für das Mammakarzinom aufzustellen, die Einsichten in die genetischen Grundlagen dieser Erkrankung erlaubt. Interessant ist, dass diese Gensignaturen letztlich die aus der klassischen Pathologie und der Immunhistochemie bekannten Befunde bestätigt haben, ja sogar teilweise erst zu einem Bewusstwerden und zu einer Akzeptanz bestimmter Läsionen geführt haben. So haben z. B. Genexpressionsprofile von Mammakarzinomen den aus der Immunhistochemie seit Jahrzehnten bekannten basalzelligen Karzinomen zu einer Renaissance verholfen. 


\section{Pathologische Diagnostik im Mammographiescreening}

Der notwendige interdisziplinäre Diskurs zwischen Radiologie, Pathologie und Chirurgie im Mammographiescreening hat die Bedeutung der Pathologie radikal verändert, hier seien nur einige Schlagwörter aufgeführt. Eine besondere Rolle spielt die Diagnostik an minimal-invasiv gewonnenen Biopsien (MIB).

Ziel der MIB sind Herdbefunde, Mikroverkalkungen sowie weit seltener Architekturstörungen. Die dabei histologisch entdeckten Läsionen unterscheiden sich in Screeningprogrammen erheblich von den aus der kurativen Mammadiagnostik bekannten Befunden. Das in Deutschland aufgebaute Screeningprogramm ist das erste, das von Beginn an flächendeckend die stereotaktische vakuumassistierte Biopsietechnik zur Abklärung von Mikrokalk einsetzt. In einem gesonderten Beitrag von Hungermann et al. wird beispielhaft das Spektrum der pathologischen Diagnosen nach MIB von Mikrokalk tragenden Läsionen mit dem Spektrum der übrigen Biopsien verglichen. Im Zentrum von 2 Übersichtsarbeiten widmen sich Böcker et al. den nach wie vor problematischen frühen Läsionen der duktalen Neoplasie, nämlich der flachen epithelialen Atypie und der atypischen duktalen Hyperplasie, die im Screening einen Teil der abzuklärenden Mikrokalkbefunde verursachen und auch dem erfahrenen Mammaexperten Kopfschmerzen bereiten.

Für die interdisziplinäre Entscheidungsfindung, im Screening unbedingte Grundlage des Erfolgs, war es notwendig, einen Weg zu finden, die pathomorphologischen Ergebnisse der MIB einfach und unmissverständlich zu kommunizieren. Das Ergebnis ist die in den europäischen Guidelines veröffentlichte B-Klassifikation, die morphologische Entitäten mit ähnlichen klinischen Konsequenzen zusammenfasst. Sie stellt zusammen mit dem - ebenfalls in Kategorien ausgedrückten - radiologischen Ergebnis die Grundlage der Management- und Therapieentscheidungen der interdisziplinären Mammakonferenz dar. Die B-Klassifikation und ihr Einsatz wird ausführlich im Beitrag von Bettendorf et al. dargestellt.

\section{Pathologische Voraussetzungen für individualisierte Therapie}

Die moderne Behandlung des Mammakarzinoms ist mit Entscheidungen über ein großes Spektrum von Therapiemöglichkeiten und deren Kombinationen verbunden. Heute werden diese Entscheidungen von interdisziplinären Teams getroffen, denen stets ein Pathologe angehört.

Alle etablierten Therapieguidelines basieren zunächst auf einer Stratifikation des Risikos der Patientinnen. Die korrekte Risikobeurteilung beruht mit Ausnahme des Alters der Patientin ausschließlich auf prognostischen Faktoren, die der Pathologe erarbeitet. Ihre Qualität ist entscheidend zur Vermeidung von Unter- und Übertherapie. Hinzu kommen prädiktive Aussagen über die Wirksamkeit bestimmter Therapieformen. Sie lassen sich teils zusätzlich aus den Prognosefaktoren ableiten, teils existieren prädiktive Faktoren mit minimaler oder fehlender prognostischer Aussage. Decker et al. geben einen Überblick über die aktuelle evidenzbasierte Wertigkeit prognostischer und prädiktiver Faktoren des Mammakarzinoms, woraus zwanglos die dominierende Rolle der Pathologie auf diesem Gebiet hervorgeht.

Eine enorme Verfeinerung des pathologischen Stagings hat die Etablierung der Sentinel-Lymphknoten-Biopsie (SLNB) gebracht. Sie ist besonders im Mammographiescreening mit der Dominanz kleiner hoch differenzierter und nodal negativer Karzinome relevant für die Reduktion der Morbidität der Axilla bei negativem SLNB. In einer Übersicht von G. Cserni werden zum einen die Folgen der Einführung der SLNB einschließlich des sog. Stage-Shifting und der resultierenden Wertungs- und Vergleichsprobleme dargestellt. Zum anderen werden die Probleme der Anwendung von Definitionen spezieller Befundkonstellationen in der SLNB-Diagnostik sowie deren Reproduzierbarkeit analysiert.

In jüngerer Zeit ist der Bedarf nach zusätzlichen therapierelevanten Faktoren aus zwei Gründen größer geworden: Zum einen wird nach prädiktiven Faktoren für bestimmte "targeted“ Therapien gesucht, zum anderen soll die adjuvante medika- mentöse Therapie von nodal negativen Patientinnen auf ein wirkliches Risikokollektiv beschränkt und damit die Übertherapie der überwiegenden Mehrheit vermieden werden.

Wenngleich die endokrine Therapie mit Tamoxifen bereits seit über 15 Jahren an den positiven Hormonrezeptorbefund gebunden ist, hat der Nachweis potenzieller therapeutischer Zielmoleküle erst in den letzten Jahren als „prädiktive Pathologie“ oder "Theranostics“ rasant an Bedeutung gewonnen. Mit Hilfe von CGH- und RNA-Expressionsprofilen konnte gezeigt werden, dass Her2-positive Karzinome einen eigenständigen molekularen Weg der Karzinogenese kennzeichnen. Die Bestimmung des ERBB2/Her-2/ neu-Status ist heute essenzieller Bestandteil der Mammakarzinomdiagnostik und Voraussetzung für eine Therapie mit gegen ERBB2 gerichteten Therapeutika. Die damit verbundenen Herausforderungen für die Pathologie werden am Beispiel des ERBB2-Nachweises in dem Beitrag von Rüschhoff et al. dargestellt.

Große Hoffnungen wurden in den letzten Jahren durch erste Ergebnisse von Hochdurchsatzmethoden zur Genexpressionsanalyse geweckt. Geyer et al. werten in ihrer Übersicht kritisch, welchen Beitrag bisher erarbeitete Genexpressionsprofile derzeit zu unserem Verständnis des Mammakarzinoms und zum klinischen Management leisten. Sie betonen zum einen die aktuell noch begrenzte Relevanz, zum anderen zeigen sie Trends und Chancen für die nächste Zukunft auf.

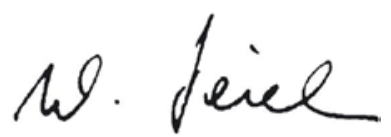

Werner Böcker

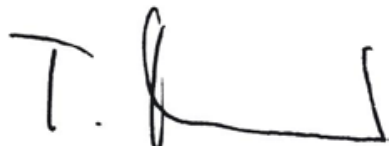

Thomas Decker

\section{Korrespondenzadresse \\ Prof. Dr. W. Böcker}

Gerhard-Domagk-Institut für Pathologie

Domagk-Straße 1, 48159 Münster

werner.boecker@ukmuenster.de 